\title{
Associação entre Sarcopenia, Estado Nutricional e Qualidade de Vida em Pacientes com Câncer Avançado em Cuidados Paliativos
}

\author{
Sarcopenia: Association with Nutritional Status and Quality of Life in Patients with Advanced-Stage Cancer in Palliative Care \\ Asociación entre Sarcopenia, Estado Nutricional y Calidad de Vida en Pacientes con Cáncer Avanzado en Cuidados \\ Paliativos
}

Mariana dos Santos Campello Queiroz ${ }^{1}$; Emanuelly Varea Maria Wiegert ${ }^{2}$; Larissa Calixto Lima²; Livia Costa de Oliveira ${ }^{2}$

\begin{abstract}
Resumo
Introduçáo: A sarcopenia pode comprometer a qualidade de vida de indivíduos com câncer. Objetivo: Avaliar a prevalência de sarcopenia e sua associação com estado nutricional e qualidade de vida em pacientes com câncer avançado em cuidados paliativos. Método: Estudo transversal com indivíduos com câncer em fase avançada, ambos os sexos, idade $>20$ anos, em seu primeiro atendimento na unidade de Cuidados Paliativos do Instituto Nacional de Câncer José Alencar Gomes da Silva. Variáveis analisadas: antropometria, força de preensão manual (FPM), Karnofsky performance status, qualidade de vida (QLQ-C15-PAL), avaliação subjetiva global produzida pelo paciente e exames laboratoriais. Para a classificação da sarcopenia, foram consideradas baixa massa muscular [circunferência muscular do braço (CMB) adequação $<90 \%$ ] e baixa força muscular [FPM <percentil 10]. Pacientes com baixa FPM foram agrupados como dinapênicos e com baixa adequação da CMB como atrofia muscular. O nível de significância estatístico adotado foi de 5\%. Resultados: Estudaram-se 210 indivíduos, média de idade $60,6( \pm 13,4)$ anos, predomínio do sexo feminino e baixa capacidade funcional. A dinapenia esteve presente em $21,9 \%$, a atrofia muscular em $17,1 \%$ e a sarcopenia em 32,4\% da amostra. Pacientes sarcopênicos apresentaram pior estado nutricional, maiores valores de leucócitos e proteína $\mathrm{C}$ reativa, e menores de linfócitos e albumina quando comparados aos não sarcopênicos. Para a qualidade de vida, foi observada diferença significativa na função física entre os grupos. Conclusáo: A sarcopenia está associada ao pior estado nutricional e pode potencializar o comprometimento funcional, reduzindo a qualidade de vida de pacientes com câncer avançado em cuidados paliativos.
\end{abstract}

Palavras-chave: Sarcopenia; Cuidados Paliativos; Estado Nutricional; Atrofia Muscular; Força Muscular.

\begin{abstract}
Introduction: Sarcopenia can compromise the quality of life of individuals with cancer. Objective: To evaluate the prevalence of sarcopenia, as well as its association with nutritional status and quality of life, among patients with advanced-stage cancer in palliative care. Method: This was a cross-sectional study involving patients with advanced-stage cancer who were $\geq 20$ years of age and were receiving treatment for the first time in the Palliative Care Unit of the José Alencar Gomes da Silva National Cancer Institute. The following variables were analyzed: anthropometric parameters; handgrip strength (HGS); Karnofsky performance status; quality of life (QLQ-C15PAL score); Patient-Generated Subjective Global Assessment score; and laboratory tests. Sarcopenia was diagnosed on the basis of low muscle mass (mid-arm circumference [MAC] adequacy <90\%) and low muscle strength (HGS $<10$ th percentile). Patients with low HGS were classified as having dynapenia, and those with low MAC adequacy were classified as having muscle atrophy. The level of statistical significance adopted was $5 \%$. Results: A total of 210 patients were studied. The mean age was $60.6( \pm 13.4)$ years. There was a predominance of females and of patients with low functional capacity. Among the patients in the sample, we identified dynapenia in $21.9 \%$, muscular atrophy in $17.1 \%$, and sarcopenia in $32.4 \%$. The patients with sarcopenia had worse nutritional status, higher leukocyte counts, higher C-reactive protein levels, lower lymphocyte proportions, and lower albumin levels than did those without sarcopenia. We observed a significant difference between those two groups in terms of physical impairment affecting quality of life. Conclusion: Sarcopenia is associated with poor nutritional status and can potentiate functional impairment, reducing the quality of life of patients with advanced-stage cancer in palliative care.

Key words: Sarcopenia; Palliative Care; Nutritional Status; Muscular Atrophy; Muscle Strength.
\end{abstract}

Resumen

Introducción: La sarcopenia puede comprometer la calidad de vida de los individuos con cáncer. Objetivo: Evaluar la prevalencia de sarcopenia y su asociación con el estado nutricional y la calidad de vida en pacientes con cáncer avanzado en cuidados paliativos. Método: Estudio transversal con individuos con cáncer avanzado, ambos sexos, edad >20 ańos, en su primera atención en la unidad de Cuidados Paliativos del Instituto Nacional de Cáncer José Alencar Gomes da Silva. Las variables analizadas fueron: antropometría, fuerza por dinamometría (FPD), Karnofsky performance status, calidad de vida (QLQC15-PAL), Valoración Subjetiva Global generada por el paciente y exámenes de laboratorio. Para una composición de sarcopenia se consideró como baja masa muscular [circunferencia muscular del brazo (CMB) adecuación $<90 \%$ y baja fuerza muscular [FPD < percentil 10]. Pacientes con Baja FPD se agruparon como dinapénicos y con baja adecuación de $\mathrm{CMB}$ como atrofia muscular. El nivel de significancia estadística adoptado fue del 5\%. Resultados: Estudió 210 individuos, media de edad 60,6 $( \pm 13,4)$ años, predominio del sexo femenino y baja capacidad funcional. La dinapenia estaba presente en el $21,9 \%$, la atrofia muscular en el $17,1 \%$ y la sarcopenia en el $32,4 \%$ de la muestra. Los pacientes sarcopénicos presentaron peor estado nutricional, mayores valores de leucocitos y proteína $C$ reactiva y menores de linfocitos y albúmina cuando comparados a los en el sarcopénico. Para la calidad de vida, fue observada diferencia en la función física entre los grupos. Conclusión: La sarcopenia está asociada a un peor estado nutricional y potencial compromiso funcional, y reduce la calidad de vida de los pacientes con cáncer avanzado en cuidados paliativos.

Palabras clave: Sarcopenia; Cuidados Paliativos; Estado Nutricional; Atrofia Muscular; Fuerza Muscular.

\footnotetext{
${ }^{1}$ Discente do Curso de Aperfeiçoamento I do Instituto Nacional de Câncer José Alencar Gomes da Silva (INCA). Rio de Janeiro (RJ), Brasil.

${ }^{2}$ Nutricionista. Unidade de Cuidados Paliativos do INCA. Rio de Janeiro (RJ), Brasil.

Endereço para correspondência: Emanuelly Varea Maria Wiegert. Rua Visconde de Santa Isabel, no 274 - Vila Isabel. Rio de Janeiro (RJ), Brasil. CEP $20560-120$. E-mail:manuvarea@gmail.com.
} 


\section{INTRODUÇÃO}

A palavra sarcopenia deriva do grego sarkós (carne) + penía (pobreza, privação) ${ }^{1}$. O termo foi empregado pela primeira vez em 1889, por Inwin Rosenberg, para descrever a diminuição da massa muscular relacionada ao envelhecimento ${ }^{2}$. Atualmente, é definida como uma síndrome clínica caracterizada pela perda progressiva e generalizada de força e massa muscular ${ }^{1}$.

Embora frequentemente observada em idosos, a sarcopenia pode ocorrer em indivíduos de qualquer faixa etária. Decorre da ingestáo inadequada de energia e/ou proteínas, má absorção intestinal, distúrbios gastrintestinais, uso de alguns medicamentos, anorexia ou ainda em consequência de determinadas doenças, entre as quais se destaca o câncer, particularmente em sua fase avançada ${ }^{3}$.

A prevalência da sarcopenia em indivíduos com tumores malignos varia conforme o método de definição empregado e pode ter como consequências diminuição da funcionalidade, pior qualidade de vida, maior tempo de hospitalização e menor sobrevida ${ }^{3,4}$. $\mathrm{Na}$ fase avançada da doença, a diminuição da função muscular é um dos indicadores de sobrevida e, quando interpretada em conjunto com outros fatores, pode oferecer informaçóes prognósticas 5 .

Ao nosso conhecimento, não há evidências científicas que tenham focado no impacto da sarcopenia sobre a qualidade de vida de indivíduos com doença avançada em cuidados paliativos. Dessa forma, o presente estudo tem como objetivo avaliar a prevalência de sarcopenia em pacientes com câncer avançado em cuidados paliativos e investigar se essa condição se associa com o estado nutricional e a qualidade de vida.

\section{MÉTODO}

Estudo transversal e analítico, proveniente de um estudo maior em andamento, realizado com pacientes com câncer avançado, avaliados em até 48 horas do primeiro atendimento na Unidade de Cuidados Paliativos (HC IV) do Instituto Nacional de Câncer José Alencar Gomes da Silva (INCA), no Rio de Janeiro.

Foram recrutados indivíduos, de ambos os sexos, diagnosticados com tumores malignos em estágio avançado, no período de julho a setembro de 2016. Os critérios de inclusão foram: idade $\geq 20$ anos; ser capaz de responder às informaçôes necessárias ou estarem acompanhados de responsável capaz de fornecê-las; Karnofsky performance status (KPS) $\geq 30 \%$; concordar em assinar o Termo de Consentimento Livre e Esclarecido
(TCLE). O estudo foi aprovado pelo Comitê de Ética em Pesquisa do INCA sob o número de protocolo CAAE: 52396416.4.0000.5274.

O peso corporal, a estatura, a circunferência do braço (CB) e a prega cutânea tricipital (PCT) foram aferidos de acordo com a metodologia proposta por Lohman et $\mathrm{al}^{6}$. O peso foi medido em quilogramas $(\mathrm{Kg})$ usando uma balança portátil Wiso Digital calibrada (capacidade de $150 \mathrm{~kg}$ ). Foi utilizada uma "cama balança" da marca Stryker, modelo Go Bed II, para pacientes acamados. A estatura foi medida usando uma fita métrica na parede. $\mathrm{Na}$ impossibilidade de mensuração, tal estatura foi estimada por meio da equação proposta por $\mathrm{Chumlea}^{7}$, que utiliza a altura do joelho. Essa medida foi realizada com as articulaçóes do joelho e tornozelo flexionados a $90^{\circ}$, utilizando-se uma fita métrica inextensível. A PCT foi obtida com a utilizaçâo de adipômetro da marca Lange ${ }^{\circ}$.

A circunferência muscular do braço (CMB) foi obtida por meio da equação proposta por Gurney; Jelliffe ${ }^{8}$. O percentual de adequação da CB, PCT e CMB foi obtido ao se comparar com os valores de referência (percentil 50) do National Health and Nutrition Examination Survey (NHANES), demonstrados em tabela de percentis por Frisancho (1981) $)^{9}$, de acordo com o sexo e a faixa etária. O índice de massa corporal (IMC) foi determinado pela divisão do peso pelo quadrado da estatura.

Foi utilizada a versão reduzida da avaliação subjetiva global produzida pelo paciente (ASG-PPP), proposta por Vigano et al. ${ }^{10}$, que avalia a alteração involuntária do peso corporal, a história alimentar, a presença de sintomas de impacto nutricional e a avaliação da capacidade funcional, gerando um escore numérico baseado no somatório de cada um dos itens do questionário (quanto mais alta é a pontuação, maior é o risco de desnutrição). Foram considerados em risco nutricional indivíduos com pontuaçáo total maior ou igual a nove pontos.

A força de preensão manual (FPM) foi avaliada a partir da medida da contração isométrica dos músculos da mão, com a utilização do dinamômetro hidráulico Jamar $^{\circ}$, que possui escala de 0 a $100 \mathrm{~kg}$ e resoluçâo de $1,0 \mathrm{~kg}$. A contração ocorreu a partir de um comando verbal do avaliador. A preensão manual foi repetida por três vezes em cada membro, estando o indivíduo sentado, com intervalos de cerca de 1 minuto, onde foi desprezada a primeira medida e registrada a maior medida das duas últimas contraçôes.

Para classificação da sarcopenia, foram utilizadas a baixa massa e a força muscular. Para o primeiro critério, foi adotada uma adequação da $\mathrm{CMB}<90 \%$. Para o segundo, adotou-se a FPM < percentil 10 conforme sexo, idade e lado do braço, utilizando como referência um estudo composto por indivíduos saudáveis da cidade de Niterói ${ }^{11}$. Os pacientes foram classificados como: sarcopênicos - 
caracterizados pela associação de baixa $\mathrm{CMB}$ e de baixa FPM; dinapênicos - baixa FPM, isoladamente; atrofia muscular - baixa CMB, isoladamente.

Para avaliação da qualidade de vida, foi empregada a versão traduzida em português do questionário QLQ-C15-PAL, desenvolvido pela European Organization for Research and Treatment of Cancer (EORTC), mediante autorização. O questionário é composto por 15 itens, contendo componentes relativos à autonomia e funcionalidade, sintomas físicos e emocionais e qualidade de vida global. Para determinaçáo da pontuação final, foram utilizadas as fórmulas propostas por Groenvold et al. ${ }^{12}$.

O KPS foi utilizado para avaliar a capacidade funcional. Os pacientes foram classificados com os valores de 30\% a $100 \%$ pelo pesquisador no momento da avaliação (100\%: função completa - 0\%: morte).

Foram avaliados hemograma completo, albumina sérica e proteína $\mathrm{C}$ reativa (PCR) ultrassensível. Tais dosagens são realizadas como rotina na unidade. A coleta de sangue foi realizada por um técnico de enfermagem, durante a consulta ambulatorial e, para os pacientes internados, em no máximo 48 horas após a internação.

Foi realizada a descriçáo da amostra segundo procedimentos clássicos como média e desvio-padrão (DP) ou mediana e limites (mínimo e máximo), para variáveis contínuas simétricas e assimétricas, respectivamente; e como número de observaçóes (n) e frequência (\%) para variáveis categóricas. $\mathrm{O}$ teste Kolmogorov-Smirnov foi utilizado para avaliar a simetria de distribuição das variáveis. A comparação entre dois grupos foi realizada por meio do teste $t$ de Student não pareado para as variáveis com distribuição normal ou pelo teste não paramétrico correspondente, Mann-Whitney-Wilcoxon, para as variáveis com distribuiçãa não normal. O software Statistical Package for the Social Sciences (SPSS versão 21.0; Chicago, IL) foi utilizado para realizar os testes estatísticos. A probabilidade de significância aceita foi de $p$ valor menor que 0,05 .

\section{RESULTADOS}

Participaram do estudo 210 indivíduos, com média de idade em torno da sexta década de vida e predomínio do sexo feminino. Os sítios tumorais mais prevalentes foram os do trato gastrintestinal (TGI), seguido pelos tumores ginecológicos (útero, endométrio e vulva). Aproximadamente três quartos dos pacientes possuíam capacidade funcional reduzida $\left(\mathrm{KPS}_{\leq} 50 \%\right)$. A média do escore total da versão reduzida da ASG-PPP foi indicativa de risco nutricional (Tabela 1 ).

Observou-se que a dinapenia esteve presente em $21,9 \%$, a atrofia muscular em $17,1 \%$ e a sarcopenia, caracterizada pela associação de ambas as condições, em $32,4 \%$ da amostra estudada (Figura 1).
Tabela 1. Características gerais dos pacientes com câncer avançado atendidos em uma Unidade de Cuidados Paliativos da cidade do Rio de Janeiro

\begin{tabular}{l|c}
\multicolumn{1}{c|}{ Variáveis } & n (\%) \\
\hline Idade (anos) & $60,6( \pm 13,4)^{\mathrm{a}}$ \\
\hline Sexo feminino & $134(63,8)$ \\
\hline Cor/Raça & $82(39,1)$ \\
\hline Branca & $75(35,7)$ \\
\hline Parda & $53(25,2)$ \\
\hline Preta & \\
\hline Localização tumoral & $56(26,7)$ \\
\hline TGI & $42(20,0)$ \\
\hline Ginecológico & $30(14,3)$ \\
\hline Mama & $26(12,4)$ \\
\hline Cabeça e pescoço & $24(11,4)$ \\
\hline Tórax & $5(2,4)$ \\
\hline Próstata & $27(12,9)$ \\
\hline Outros & \\
\hline Tratamento prévio & $84(40,0)$ \\
\hline Cirurgia & $132(62,9)$ \\
\hline Quimioterapia & $97(46,2)$ \\
\hline Radioterapia & $13,4(7,5 ; 19,0)^{\mathrm{b}}$ \\
\hline Pontuação ASG-PPP & $161(76,7)$ \\
\hline KPS 30-50\% &
\end{tabular}

n=número de observaçōes; \%=frequência; TGI=trato gastrintestinal; ASG$\mathrm{PPP}=$ avaliaçáo subjetiva global produzida pelo paciente; KPS=Karnofsky performance status.

${ }^{2}$ Média \pm desvio-padráo

'Mediana (mínimo e máximo)

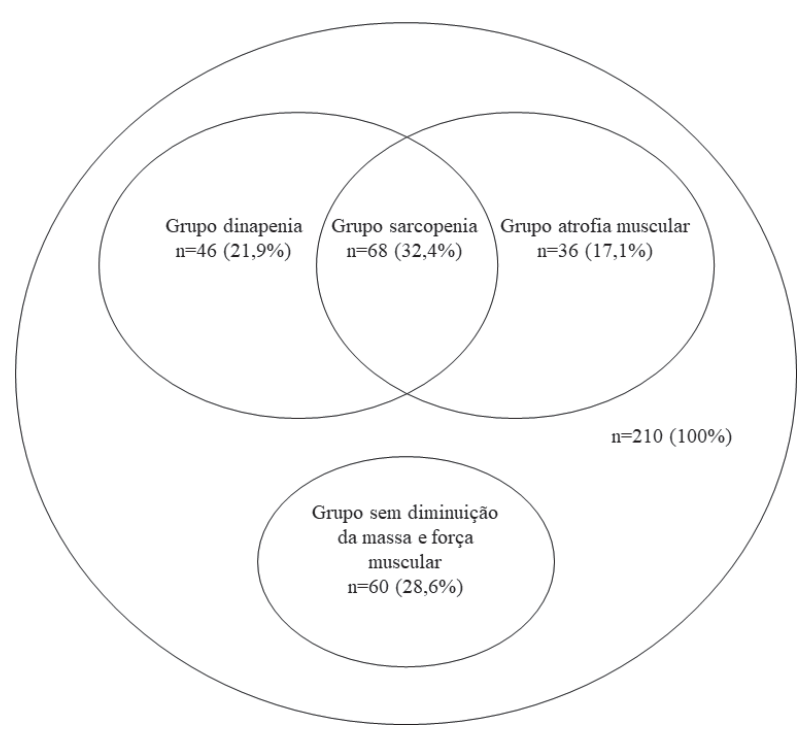

Figura 1. Prevalência de dinapenia, atrofia muscular e sarcopenia em pacientes com câncer avançado atendidos em uma Unidade de Cuidados Paliativos da cidade do Rio de Janeiro 
Os valores séricos dos leucócitos, linfócitos, PCR e albumina foram significativamente diferentes entre os grupos sarcopênicos e não sarcopênicos. No que se refere ao estado nutricional, observa-se que todas as variáveis antropométricas foram significativamente menores nos indivíduos sarcopênicos que, além disso, também apresentaram maior pontuação total da ASG-PPP e em seus domínios relativos à história de perda de peso e capacidade funcional (Tabela 2).

Os parâmetros concernentes à qualidade de vida seguem descritos na Tabela 3. Das quatro dimensóes avaliadas, nomeadamente física, emocional, sintomas e saúde global, a primeira foi a única que apresentou diferença significativa entre os grupos, indicando que indivíduos sarcopênicos possuíam funcionalidade prejudicada.

\section{DISCUSSÃO}

Os principais achados do presente estudo foram: prevalência de $32,4 \%$ de sarcopenia, somado ao fato de que cerca de metade dos indivíduos apresenta prejuízo na força ou atrofia muscular; marcadores inflamatórios e nutricionais alterados entre os sarcopênicos; e, por fim, menor funcionalidade entre os indivíduos desse mesmo grupo.

Foi utilizada, como critério diagnóstico de sarcopenia, a diminuição da massa e da força muscular ${ }^{1}$. Não foram encontradas publicações na literatura científica que avaliassem a prevalência de sarcopenia em pacientes com câncer avançado utilizando os mesmos critérios e métodos de avaliação. Entretanto, estudos semelhantes, que utilizaram como critério apenas a baixa massa muscular, encontraram uma prevalência ainda mais elevada. A exemplo, Rollins et al. ${ }^{13}$, ao avaliar a composição corporal utilizando tomografia computadorizada (TC), encontraram, em uma amostra de 228 indivíduos com câncer de pâncreas avançado, prevalência de 60,5\% de sarcopenia. No estudo de Paireder et al. ${ }^{14}$, a prevalência foi de 61,5\% em 130 indivíduos com tumores esofágicos com a utilização do mesmo método diagnóstico.

A TC consiste no método padrão-ouro para a avaliação da massa muscular. Apresenta, porém, a limitação de

Tabela 2. Características laboratoriais e de estado nutricional de acordo com presença de sarcopenia em pacientes com câncer avançado atendidos em uma Unidade de Cuidados Paliativos da cidade do Rio de Janeiro

\begin{tabular}{|c|c|c|c|}
\hline Variáveis & $\begin{array}{c}\text { Sarcopênicos } \\
(n=68)\end{array}$ & $\begin{array}{c}\text { Não sarcopênicos } \\
(n=142)\end{array}$ & $\boldsymbol{p}^{a}$ \\
\hline Hemácias $\left(3,9-5,6\right.$ milhões/dL) ${ }^{b}$ & $3,7( \pm 0,8)$ & $3,8( \pm 0,8)$ & 0,683 \\
\hline Hemoglobina $(11,5-16,4 \mathrm{~g} / \mathrm{dL})^{c}$ & $10,0(8,2 ; 12,1)$ & $10,7(9,0 ; 11,9)$ & 0,159 \\
\hline Hematócrito (36-47\%) ${ }^{b}$ & $32,6( \pm 6,2)$ & $30,8( \pm 7,2)$ & 0,099 \\
\hline Leucócitos $(4.000-10.000 / \mathrm{mm} 3)^{c}$ & $9.700(7.200 ; 15.800)$ & $8.600(5.900 ; 11.750)$ & 0,044 \\
\hline Linfócitos $(20-45 \%)^{c}$ & $10(5,0 ; 18,4)$ & $14,4(9,1 ; 22,2)$ & 0,002 \\
\hline $\operatorname{PCR}(<0,5 \mathrm{mg} / \mathrm{dL})^{c}$ & $6,28(2,7 ; 14,8)$ & $4,8(1,5 ; 11,8)$ & 0,048 \\
\hline Albumina $(3,5-5,2 \mathrm{~g} / \mathrm{dl})^{\mathrm{c}}$ & $2,9(2,3 ; 3,6)$ & $3,4(2,9 ; 4,0)$ & 0,011 \\
\hline Peso $(\mathrm{Kg})^{c}$ & $47,1(41,1 ; 55,7)$ & $62,8(53,8 ; 74,1)$ & 0,0001 \\
\hline $\operatorname{IMC}\left(\mathrm{Kg} / \mathrm{m}^{2}\right)^{\mathrm{c}}$ & $18,2(15,8 ; 21,1)$ & $24,1(20,5 ; 29,0)$ & 0,0001 \\
\hline Adequação de $\mathrm{CB}^{\mathrm{c}}$ & $69,5(61,2 ; 76,2)$ & $91,8(81,7 ; 103,6)$ & 0,0001 \\
\hline Adequação de $\mathrm{PCT}^{\mathrm{c}}$ & $45,5(32,2 ; 72,4)$ & $78,7(49,6 ; 103,9)$ & 0,0001 \\
\hline Adequação de $\mathrm{CMB}^{\mathrm{c}}$ & $77,9(70,4 ; 83,6)$ & $98,1(90,7 ; 105,3)$ & 0,0001 \\
\hline Dinamometria $^{b}$ & $19,71( \pm 10,7)$ & $13,22( \pm 7,7)$ & 0,0001 \\
\hline Pontuação ASG-PPPc & $14,5(11,0 ; 19,0)$ & $13,0(7,25 ; 17,0)$ & 0,025 \\
\hline \multicolumn{4}{|l|}{ Domínios ASG-PPPc } \\
\hline História de perda de peso ${ }^{c}$ & $4,0(1,0 ; 5,0)$ & $2,0(0 ; 1,0)$ & 0,013 \\
\hline Alteração da ingestão alimentar & $0(0 ; 2,0)$ & $1,0(0 ; 1,0)$ & 0,407 \\
\hline Sintomas de impacto nutricionalc & $9(5,0 ; 13,0)$ & $7,0(3,2 ; 11,0)$ & 0,061 \\
\hline Capacidade funcionalc & $3(3,0)$ & $3,0(1,0 ; 3,0)$ & 0,003 \\
\hline
\end{tabular}

PCR=proteína $C$ reativa; IMC=índice de massa corporal; $\mathrm{CB}=$ circunferência do braço; $\mathrm{PCT}$ =prega cutânea tricipital; $\mathrm{CMB}=$ circunferência muscular do braço; ASG-PPP=avaliação subjetiva global produzida pelo paciente, versão resumida.

${ }^{a}$ Teste $t$ Student para amostras independentes ou teste de Mann Whitney $\mathrm{U}$, conforme apropriado.

${ }^{\mathrm{b}}$ Média \pm desvio-padrão.

‘Mediana (mínimo e máximo) 
Tabela 3. Dimensões da qualidade de vida de acordo com o estado nutricional em pacientes com câncer avançado atendidos em uma Unidade de Cuidados Paliativos da cidade do Rio de Janeiro

\begin{tabular}{|c|c|c|c|}
\hline Variáveis & $\begin{array}{l}\text { Sarcopênicos } \\
\qquad(n=68)^{a}\end{array}$ & $\begin{array}{l}\text { Não sarcopênicos } \\
(n=142)\end{array}$ & $p^{b}$ \\
\hline Físico & $78(56 ; 100)$ & $67(33 ; 89)$ & 0,012 \\
\hline Emocional & $50(0 ; 83)$ & $50(17 ; 83)$ & 0,444 \\
\hline \multicolumn{4}{|l|}{ Sintomas } \\
\hline Fadiga & $50(33 ; 100)$ & $50(33 ; 83)$ & 0,286 \\
\hline Náusea & $33(0 ; 100)$ & $33(0 ; 67)$ & 0,354 \\
\hline Dor & $67(33 ; 100)$ & $67(17 ; 100)$ & 0,345 \\
\hline Dispneia & $0(0 ; 67)$ & $0(0 ; 67)$ & 0,852 \\
\hline Insônia & $67(0 ; 100)$ & $33(0 ; 100)$ & 0,714 \\
\hline Apetite & $33(0 ; 100)$ & $67(0 ; 100)$ & 0,296 \\
\hline Constipação & $0(0 ; 100)$ & $67(0 ; 100)$ & 0,164 \\
\hline Saúde global & $50(17 ; 67)$ & $67(33 ; 83)$ & 0,125 \\
\hline
\end{tabular}

$\mathrm{n}=$ número de observaçóes.

${ }^{\text {a } I I Q=I n t e r v a l o ~ i n t e r q u a r t i ́ l i c o . ~}$

b Teste Mann Whitney U.

expor o paciente à radiaçáo, constituindo-se, portanto, um exame de conveniência ${ }^{4}$. A CMB, em contrapartida, fornece uma estimativa da reserva proteica somática, sendo considerado um método simples, não invasivo, objetivo, de baixo custo e que não requer nenhuma preparaçáo ou desconforto para o paciente ${ }^{15}$. Ao avaliar várias características clínicas de pacientes com câncer avançado, Tartari et al. ${ }^{15}$ observaram que a CMB pode ser uma ferramenta útil para indicar baixa massa muscular, bem como mostrado no presente estudo.

Estudos demonstraram que a massa muscular não é linearmente relacionada com a função muscular e que esta última é melhor preditora de desfechos adversos quando comparada com a primeira ${ }^{16}$. Tais achados levaram à inclusão da função muscular na definição de sarcopenia. Nesse contexto, a FPM pode ser considerada um indicador válido do estado geral de saúde ${ }^{17}$ e a não inclusão dessa medida no diagnóstico de sarcopenia pode estimar erroneamente a sua prevalência. $\mathrm{O}$ estudo de Kilgour et $\mathrm{al}^{18}{ }^{18}$ demostrou que a baixa FPM (< percentil 10) foi preditor independente para a sobrevida (hazard ratio - HR) 3,2; intervalo de confiança (IC) 95\%:2,0-5,1) e associada à maior presença de sarcopenia (odds ratio - OR, 9,53; IC95\%: 1,95-46,55), além de alteraçóes biológicas e funcionais e de pior qualidade de vida em pacientes com câncer avançado.

O diagnóstico precoce e adequado é importante para que os efeitos da sarcopenia possam ser atenuados ou adiados por meio de intervenção nutricional apropriada o mais brevemente possível durante a trajetória da doença.

Nossos resultados demostraram que indivíduos sarcopênicos apresentaram níveis séricos de PCR e de leucócitos mais elevados, bem como de linfócitos e albumina reduzidos quando comparados com os não sarcopênicos. Sabe-se que a inflamação sistêmica está associada ao aumento do catabolismo proteico, levando à diminuição da massa muscular. Nossos achados corroboram os de Kim et al. ${ }^{19}$, que, estudando 186 indivíduos com câncer de pulmão, encontraram níveis séricos de PCR e contagem de leucócitos mais elevados no grupo com sarcopenia.

No que concerne ao estado nutricional, todos os marcadores avaliados foram piores no grupo com sarcopenia. Apoiando esses achados, Zhou et al. ${ }^{20}$, em um estudo prospectivo incluindo pacientes com câncer gástrico, também demostraram que indivíduos sarcopênicos apresentavam menor IMC e níveis mais baixos de albumina sérica. De forma similar, Kim et al. ${ }^{19}$ avaliaram pacientes com câncer de pulmão de pequenas células e encontraram que a sarcopenia associou-se de forma significativa com IMC, albumina sérica e peso corporal. Cabe destacar que a utilização dos marcadores antropométricos, assim como os níveis séricos de albumina e a pontuação da ASG-PPP foram sensíveis para identificar o comprometimento do estado nutricional em indivíduos sarcopênicos. Esses achados ressaltam que a utilização de instrumentos simples e de fácil aplicabilidade são satisfatórios para identificação precoce da desnutrição associada à sarcopenia.

Sobre a qualidade de vida, os resultados demonstraram diferenças significativas apenas no domínio referente ao comprometimento físico, mostrando que a baixa força e a perda de massa muscular levam a uma deterioraçáo da função física e autonomia, observada na dificuldade em realizar atividades de vida diárias. Segundo o estudo de Frio et al. ${ }^{21}$, os pacientes que possuem diminuição da massa muscular apresentam escores mais baixos em 
qualidade de vida, comparados aqueles sem déficit de massa muscular. Uma redução na massa muscular pode influenciar na função muscular e levar à perda de força, reduçáo da funçáo pulmonar, aumento da incapacidade e, portanto, pior qualidade de vida ${ }^{18}$. No entanto, de acordo com Van Roekel et al. ${ }^{22}$, a sarcopenia não se associou à qualidade de vida em pacientes com câncer colorretal em estágios I-III. Na coorte de Bye et al. ${ }^{23}$, foi verificado que a massa muscular tinha que estar abaixo de um determinado valor antes de impactar negativamente na função física e, mais ainda, em outros indicadores de qualidade de vida, o que ajuda a explicar nossos achados.

O presente estudo apresenta limitaçóes. Em primeiro lugar, métodos precisos como TC e densitometria de dupla energia de raios X (DXA) náo foram utilizados para avaliar a massa muscular. Segundo, pela falta de padrão de referência de parâmetros antropométricos e de FPM específicos para indivíduos com câncer avançado em cuidados paliativos. Por outro lado, cabe destacar que foram empregadas medidas de massa muscular de baixo custo e de fácil utilização para critérios diagnósticos de sarcopenia. Esses métodos são necessários para a triagem, particularmente nos países em desenvolvimento, uma vez que os métodos padrão-ouro utilizados são financeiramente inviáveis em larga escala.

\section{CONCLUSÃO}

A sarcopenia está associada ao pior estado nutricional e pode potencializar o comprometimento funcional, comumente presente em indivíduos com câncer avançado. Sendo assim, a uniformização dos critérios diagnósticos e a elaboração de protocolos de assistência nutricional específicos para essa população podem refletir em melhora da qualidade de vida, principal objetivo dos cuidados paliativos.

\section{CONTRIBUIÇÕES}

Mariana dos Santos Campello Queiroz, Emanuelly Varea Maria Wiegert, Larissa Calixto Lima e Livia Costa de Oliveira participaram do planejamento do estudo, da coleta e da análise dos dados e revisaram e aprovaram a revisão final.

\section{DECLARAÇÃO DE CONFLITO DE INTERESSES}

Nada a Declarar.

\section{REFERÊNCIAS}

1. Santilli V, Barnetti A, Mangone M, Paoloni M. Clinical definition of sarcopenia. Clinical Cases in Mineral and Bone Metabolism. 2014; 11(3):177-180.
2. Cruz-Jentoft, AJ, Baeyens PJ, Bauer MJ, Boirie, Y, Cederholm, T, et al. Sarcopenia: European consensus on definition and diagnosis. Report of the European Working Group on Sarcopenia in Older People. Age and Ageing. 2010; 39(4):412-423.

3. Collins JT, Noble S, Chester J, Davies HE, Evans WD, et al. Association of sarcopenia and observed physical performance with attainment of multidisciplinary team planned treatment in non-small cell lung cancer: an observational study protocol. BMC Cancer. 2015;15(544):1-6.

4. Prado CMM, Baracos VE, Mccargar LJ, Reiman T, Mourtzakis M, Tonkin K, et al. Sarcopenia as a Determinat of Chemetherapy Toxicity and Time to Tumor Profression in Metastatic Breast Cancer Patients Receiving Capecitabine Treatment. Clinical Cancer Research. 2009; 15(8):2920-6.

5. Salpeter SR, Malter DS, Luo EJ, Lin AY, Stuart B. Systematic Review of cancer presentations with a median survival of six months or less. Journal of Palliative Medicine. 2012; 15(2):175-185.

6. Lohman OTG, Roche AF, Martorell R. Anthropometric standardization reference manual [Internet]. Champaign: Human Kinetics Books, 1988 [acesso 2018 jun 23]. Disponível em: http://www.tandfonline.com/doi/abs/1 $0.1080 / 00140138808966796$ ? journalCode $=\operatorname{terg} 20$

7. Chumlea WC, Guo S, Steinbaugh ML. Prediction of stature from knee height for black and white adults and children with application to mobility-impaired or handicapped persons. J Am Diet Assoc. 1994; 94(12):1385-91.

8. Gurney, JM, Jeliffe DB. Arm anthropometry in nutritional as assessment: monogram for rapid calculation of muscle circumference and cross-section al muscle and fat areas. The American Journal of Clinical Nutrition. 1973; 26(9): 912-5.

9. Frisancho AR. New norms of upper limb fat and muscle areas for assessment of nutritional status. American Journal of Clinical Nutrition. 1981; 34(11): 2540-5.

10. Vigano, A.L.; Tomasso, J.; Kilgour, R.D.; Trutschnigg, B.; Lucas, E.; Morais, J.A; Borod, M. The abridged patientgenerated subjective global assessment is a useful tool for early detection and characterization of cancer cachexia. Journal of the Academy of Nutrition and Dietetics. 2014; 114(7):1088-98.

11. Schlussel, MM, Anjos LA, Vasconcellos MT, Kac, G. Reference values of handgrip dynamometry of healthy adults: a population-based study. Clin Nutr. 2008; 27:601-7.

12. Groenvold M, Petersen MA, Aaronson NK, Arraras JL, Blazeby JM, Boyyomley A, et al. The development of the 
EORTC QLQ-C15-PAL: a shortened questionnaire for cancer patients in palliative care. European Journal of Cancer. 2006; 42(1):55-64.

13. Rollins KE, Tewari N, Ackner A, Awwad, A, Madhusudan $S$, Macdonald, IA, et al. The impact of sarcopenia and myosteatosis on outcomes of unresectable pancreatic cancer or distal cholangiocarcinoma. Clin Nutr. 2016; 35(5):1103-9.

14. Paireder, M.; Asari, R.; Kristo, I.; Rieder, E.; Tamandl, D.; Ba-Ssalamah, A. et al. Impact of sarcopenia on outcome in patients with esophageal resection following neoadjuvant chemotherapy for esophageal cancer. Eur J Surg Oncol. 2017; 43(2):478-484.

15. Tartari RF, Ulbrichkulczynski, JM, Ferreira Filho A. Medição da circunferência muscular do meio do braço e prognóstico em pacientes com câncer de pulmão de células não pequenas de estágio IV. Oncol Lett. 2013; 5(3):1063-67.

16. Newman, AB, Kupelian V, Visser M, Simonsick EM, Goodpaster BH, Kritchevsky, S.B, et al. Strength, but not muscle mass, is associated with mortality in the health, aging and body composition study cohort. J Gerontol A Biol Sci Med Sci. 2006; 61(1):72-7.

17. Nordenskiöld UM, Grimby G. Força de preensão em pacientes com artrite reumatóide e fibromialgia e em indivíduos saudáveis. Um estudo com o instrumento Grippit. Scand J Rheumatol. 1993; 22:14-9.

18. Kilgour, RD, Vigano A, Trutschnigg B, Lucar E, Borod M.; Morais JA. Handgrip strength predicts survival and is associated with markers of clinical and functional outcomes in advanced cancer patients. Support Care Cancer. 2013; 21(12):3261-3270.

19. Kim EY, Kim YS, Seo JY, Park I, Ahn HK, Jeong, YM. The Relationship between Sarcopenia and Systemic Inflammatory Response for Cancer Cachexia in Small Cell Lung Cancer. PloS ONE. 2016; 11(8): e0161125.

20. Zhou CJ, Zhang FM, Zhang FY, Yu Z, Chen, XL, Shen $X$, et al. Sarcopenia: a new predictor of postoperative complications for elderly gastric cancer patients who underwent radical gastrectomy. J Surg Res. 2017; 211:137-146.

21. Frio CC, Pretto ADB, Gonzalez MC, Pastore CA. The Influence of Body Composition on Cancer Patients' Quality of Life. Revista Brasileira de Cancerologia. 2015; 61(4):351-357.

22. Van Roekel, EH, Bours MJL, Molder EM, BreedveldPeters JJL, Damink SWMO, Schouten LJ, et al. Associations of adipose and muscle tissue parameters at colorectal cancer diagnosis with long-term health-related quality of life. Qual Life Res. 2017; 26(7):1745-1759.
23. Bye A, Sjøblom B, Wentzel-Larsen T, Grønberg BH, Baracos VE, Hjermstad MJ, et al. Muscle mass and association to quality of life in nonsmall cell lung cancer patients. Journal of Cachexia, Sarcopenia and Muscle. 2017; 8:759-767. 